La utilidad práctica de la teoría relacional de Pierre Bourdieu en la investigación...

\title{
LA UTILIDAD PRÁCTICA DE LA TEORÍA RELACIONAL DE PIERRE BOURDIEU EN LA INVESTIGACIÓN SOCIOJURÍDICA*
}

\author{
Angélica Cuéllar Vázquez \\ Universidad Autónoma de MéXico
}

\section{Resumen}

En este texto se pretende ofrecer una propuesta para el uso de los conceptos de la teoría relacional del sociólogo francés Pierre Bourdieu en la investigación sociojurídica. Estas nociones son el habitus (esquemas de interpretación), campo y capital. Para lograr ese objetivo, se hará una revisión sucinta de ellas y después se expondrá su utilidad práctica en una investigación empírica que versa sobre el cambio en el modelo de justicia en México: el tránsito del sistema inquisitivo al acusatorio-oral, concretamente en la ciudad de Cuernavaca en el estado de Morelos. Por razones de espacio, solo se desarrollarán dos temas de análisis de las prácticas de los jueces: la construcción de la verdad jurídica y las nuevas y viejas prácticas, el llamado sistema "tradicio-oral".

Palabras clave: Sociología, jurista, interpretación, Derecho

La autora: profesora titular de la Facultad de Ciencias Políticas y Sociales de la Universidad Nacional Autónoma de México. Doctora en Sociología. Dirección postal: Avenida Universidad 3000, Cd. Universitaria, Coyoacán, 04510 Ciudad de México, D. F., México. Correo electrónico: acuellarunam@gmail.com

Recibido: 5 de febrero de 2015; evaluado: 15 de marzo de 2015; aceptado: 20 de abril de 2015.

Artículo de investigación que forma parte de un trabajo más extenso, apoyado por la Dirección General de Asuntos del Personal Académico (DGAPA) de la UNAM. Pertenece al Programa de apoyo a proyectos de investigación e innovación tecnológica (PAPIIT) IN 305312 y se titula "El sistema de justicia en México. Reforma y prácticas sociales". Agradezco la colaboración de Ivonne Roldán y Alhondra Rodríguez, ayudantes de investigación del Consejo Nacional de Ciencia y Tecnología (Conacyt) en la realización de este artículo.

NOVUM JUS • ISSN: 1692-6013 • Volumen 9 Nº 1 • Enero - Junio 2015 • Págs. 103-122 


\title{
THE PRACTICAL UTULITY OF PIERRE BOURDIEU'S RELATIONAL THEORY IN SOCIO-LEGAL RESEARCH*
}

\author{
Angélica Cuéllar Vázquez \\ Universidad Autónoma de MéXico
}

\begin{abstract}
The purpose of this text is to offer a proposal for the use of the concepts included in French sociologist Pierre Bourdieu's relational theory in the field of socio-legal research. Bourdieu defines such notions as habitus (schemes of interpretation), field and capital. To do this, we undertake a succinct review of the concepts and then expose their practical use in an empirical research study on the changes in the Mexican legal model, i.e., the transition from an inquisitorial system to an adversarial oral one, concretely in the city of Cuernavaca in the state of Morelos. Due to space limitations, we only develop two topics analyzing the practices and the judges: the construction of legal truth and the new and old practices, known as the oral accusatory system.
\end{abstract}

Key words: Sociology, jurist, interpretation, Law

About the author: Titular Professor of the School of Political and Social Sciences at Universidad Nacional Autónoma de México. PhD. In Sociology. E-mail: acuellarunam@gmail.com

Received: February 5, 2015; reviewed: March 15, 2015; accepted: April 20, 2015.

Research article that is part of a longer piece of work sponsored by the Directorate General for Academic Personnel Issues (DGAPA) at UNAM. The work belongs to the Technical Research and Innovation Projects Support Program (PAPIIT) IN 305312 and is entitled "El sistema de justicia en México. Reforma and prácticas sociales". I would like to thank the collaboration of Ivonne Roldán and Alhondra Rodríguez, research assistants from the National Council of Science and Technology (Conacyt) in writing this article.

NOVUM JUS • ISSN: 1692-6013 • Volumen 9 Nº 1 • Enero - Junio 2015 • Págs. 103-122 
La utilidad práctica de la teoría relacional de Pierre Bourdieu en la investigación...

\section{Introducción}

El objetivo de este texto será mostrar, con el uso de los conceptos clave de Pierre Bourdieu, cómo fueron resignificadas las prácticas de los jueces en los juicios orales a partir de la incorporación de nuevas habilidades, así como su rol en el nuevo sistema de justicia, la afectación a la nueva estructura por la forma en que incorpora nuevas prácticas y conserva antiguas inercias. En pocas palabras, quiero mostrar cómo la oralidad toca las viejas prácticas y cómo genera nuevas. Para la investigación se utilizaron técnicas de metodología cualitativa, entrevistas a los operadores jurídicos, la observación de las audiencias in situ y videos de las mismas. Las entrevistas se hicieron en los meses de enero a marzo de 2014. Los operadores entrevistados fueron seis jueces (que no cumplen un rol específico, sino rotativo, es decir, cumplen las tres funciones que establece la reforma, en procesos distintos), tres abogados defensores de oficio y tres Ministerios Públicos.

\section{La reforma en materia penal en el estado de Morelos}

La reforma al sistema de justicia penal en México fue publicada en el Diario Oficial de la Federación el 18 de junio de 2008 y trajo consigo una reestructuración completa al sistema de justicia. Con esta reforma se modificaron diversos aspectos del proceso penal:

- Seguridad pública (cuerpos de Policía).

- Procuración de justicia (funciones del Ministerio Público).

- Administración de justicia (funciones de los jueces).

En esta investigación me enfocaré en el análisis de las nuevas prácticas de la administración de justicia, o sea, las funciones que los jueces desempeñan en los juicios orales. Esta revisión se hizo tras observar cómo las nuevas prácticas que establece la reforma para el rol del juez son adaptadas y adoptadas en el nuevo contexto.

En el nuevo sistema de justicia se propone que los juicios orales sean una forma de exponer y debatir un caso con base en el principio de presunción de inocencia. El proceso es un diálogo abierto entre los operadores (jueces, Ministerios Públicos y abogados defensores) que se confrontarán para construir la verdad jurídica. El nuevo sistema de justicia instituye que la oralidad es el medio por excelencia para poner en práctica los principios rectores del sistema acusatorio y modificar las prácticas de los operadores del sistema. Los principios señalados por la reforma son:

NOVUM JUS • ISSN: 1692-6013 • Volumen 9 Nº 1 • Enero - Junio 2015 • Págs. 103-122 
- Publicidad.

- Inmediación.

- Continuidad.

- Concentración.

- Contradicción.

Como ya se mencionó, el eje de esta investigación es identificar las nuevas prácticas a partir de la oralidad. A pesar de que no se encuentra como uno de los principios establecidos en la reforma, se explica que esta es la guía de todo el curso del nuevo sistema de justicia. Para fines de la investigación sociojurídica, la oralidad representa un espacio rico para el análisis, porque significa el cambio de paradigma en las prácticas de los operadores jurídicos y consiste en pasar de una justicia escrita a una justicia oral. En el antiguo sistema inquisitivo se privilegiaban la prueba testimonial y el expediente y existía una concentración de funciones en todos los momentos del proceso en el Ministerio Público; en contraste, el nuevo sistema de justicia plantea que debe haber una separación de funciones, es decir, debe existir un equilibrio procesal, con la finalidad de dar mayor transparencia al proceso y respetar los derechos humanos de las víctimas y los imputados.

La reforma representa una modificación fundamental a las funciones de los operadores jurídicos. La capacitación ha sido gradual y ha estado a cargo de la Secretaría técnica del Consejo de coordinación para la implementación del sistema de justicia penal (Setec), que es un organismo gubernamental dependiente de la Secretaría de Gobernación. Cabe señalar que, para la capacitación de los operadores, esta Secretaría ha contado con el apoyo de organismos nacionales e internacionales como US Agency for Interntional Development (Usaid), ${ }^{1}$ el Programa de apoyo al Estado de Derecho en México (Proderecho), la Comisión nacional de tribunales superiores de justicia de los Estados Unidos Mexicanos (Conatrib) y las universidades públicas del estado de Morelos, así como diversas instituciones de España, Alemania, Chile, Canadá, Italia, Reino Unido y la Unión Europea, los cuales han invertido dinero para la implementación de la reforma.

El tránsito del sistema inquisitivo al sistema acusatorio-oral en México ha sido progresivo y, en algunos estados de la República, casi nulo. Por esa razón, el aná-

Usaid es una agencia estadounidense que se ha encargado de la capacitación a los operadores jurídicos en América Latina durante la transición a los nuevos sistemas de justicia. Además, inyecta capital a los proyectos de desarrollo de políticas estatales en las regiones de América. 
La utilidad práctica de la teoría relacional de Pierre Bourdieu en la investigación...

lisis se hizo en la ciudad de Cuernavaca. ${ }^{2}$ El estado de Morelos implementó los juicios orales casi a la par de la publicación de la reforma. Hasta este momento, los operadores jurídicos llevan siete años desempeñándose en el nuevo sistema.

En la reforma se establece un nuevo rol para todos los operadores jurídicos, mas en este artículo solo mencionaré el de los jueces, servidores públicos que pertenecen al poder judicial encargados de dirigir la presentación de los argumentos y las pruebas de manera pública, contradictoria y oral.

En los juicios orales, los jueces tienen diferentes funciones: sus decisiones en las audiencias son colegiadas, porque participan tres jueces y las sentencias deben ser dictadas de inmediato con los plazos que define la ley. Además, cada etapa del proceso cuenta con una figura de juez distinta: juez de control o garantía, encargado de vigilar que se respete el debido proceso en la etapa de investigación; el juez de juicio oral, que tiene a su cargo la emisión de la sentencia con respecto al desahogo de pruebas de las partes y el juez de ejecución, quien vigila que se cumpla la sanción impuesta.

El Artículo 20 constitucional señala, de manera general, las nuevas funciones que tienen que cumplir los jueces y su rol institucional: deben estar presentes en todo el proceso y no conocerán del caso previamente; dirigirán el desahogo y la presentación de pruebas durante la audiencia pública y oral; no pueden reunirse con ninguna de las partes sin que la otra esté presente; serán los únicos autorizados para conceder una orden de cateo u ordenar la prisión preventiva y deben aplicar los principios constitucionales que rigen el sistema para respetar el debido proceso y proteger los derechos humanos de las víctimas y los imputados.

Para establecer un perfil integral de los operadores jurídicos, en este caso los jueces, no solo es necesario contar con el perfil institucional, sino definir un perfil sociodemográfico de estos en el estado de Morelos. La unión de ambos proporciona la información suficiente para acercarnos a la caracterización del nuevo campo y de las nuevas prácticas de los jueces del sistema de justicia en México e identificar algunas de las prácticas resignificadas por ellos.

Según datos de la Setec, hasta finales de 2014 la implementación de la reforma en los estados de la República mexicana se encontraba de la siguiente manera: etapa inicial, 1; etapa de planeación, 15; etapa de entrada en vigencia, 5; operación parcial, 8 y operación total, 3, entre ellos, el estado de Morelos.

NOVUM JUS • ISSN: 1692-6013 • Volumen 9 Nº 1 • Enero - Junio 2015 • Págs. 103-122 
Obtuve el perfil sociodemográfico al examinar la información pública de los jueces en el estado de Morelos. En la actualidad, el estado cuenta con 45 jueces en materia penal, cuya media de edad está entre los 40 y 49 años (Gráfica 1):

\section{Gráfica 1}

Rango de edad de los jueces penales del estado de Morelos

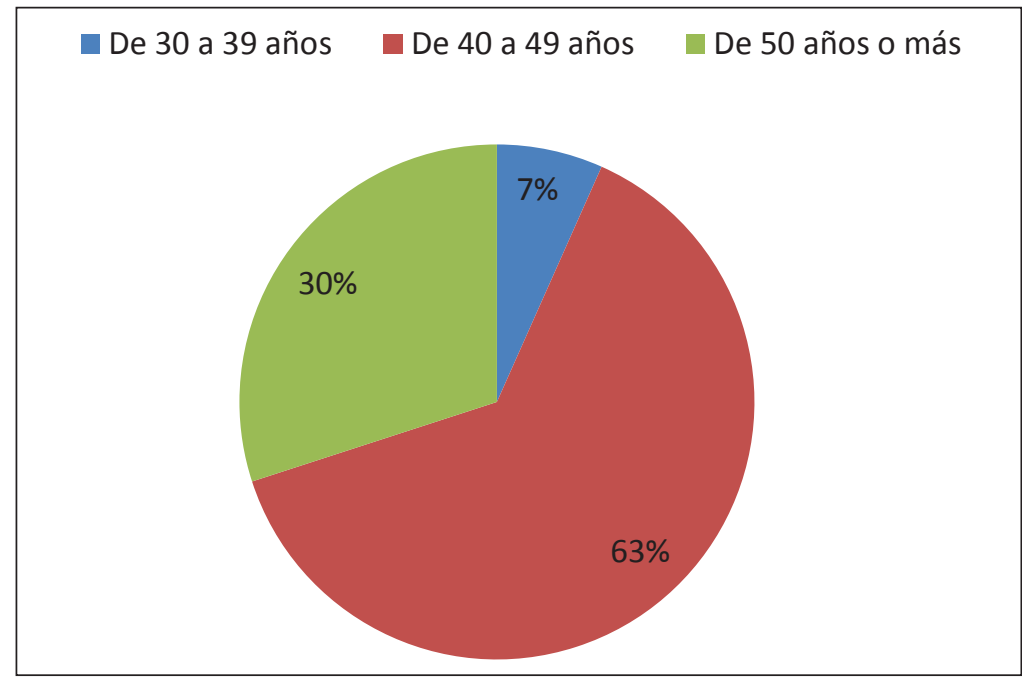

Fuente: elaboración propia

También se consideró la carrera judicial como otro elemento importante del perfil de los jueces del estado. Se observó que, antes de su nombramiento como jueces, un alto porcentaje se desempeñó como secretario de acuerdo, secretario proyectista o secretario de estudio y cuenta, pues más de $75 \%$ de los jueces actuales se desempeñó en alguna de estas funciones en el antiguo sistema.

Los secretarios proyectistas eran los encargados de leer y revisar los expedientes y, por tanto, proponer un proyecto de sentencia para que el juez lo revisara; los secretarios de acuerdo debían formar el expediente con demandas, escritos, oficios, autos y promociones que llegaban a los juzgados y los secretarios de estudio y cuenta estudiaban expedientes, demandas o escritos y presentaban un proyecto de sentencia al juez para su aprobación. Institucionalmente, los secretarios eran un apoyo para el juez; sin embargo, en la práctica, sobre ellos recaía toda la valoración del expediente, de las pruebas y la elaboración de la sentencia, lo que dio pie a que el juez no asistiera a las audiencias con regularidad. 
La utilidad práctica de la teoría relacional de Pierre Bourdieu en la investigación...

Los jueces en el estado de Morelos han recibido capacitación para operar el nuevo sistema de justicia, centrada en derechos humanos, habilidades, aptitudes y destrezas para argumentar de forma oral y también cursos para interpretar el lenguaje corporal.

\section{Las nuevas estructuras y la definición del nuevo campo sociojurídico}

En este apartado se definirá cuál es la disposición del nuevo campo del sistema de justicia en Cuernavaca a partir del uso de los conceptos de Pierre Bourdieu, en este caso, el campo, para observar cómo los jueces asumen las nuevas prácticas y resignifican el campo jurídico.

La premisa falsa de superar dualidades (macro-micro, estructura-sujeto, métodoteoría) llevó a Bourdieu a hacer una propuesta teórica relacional, es decir, entender la acción del agente no solo con base en una estructura, sino también en la observación de la influencia que el sujeto tiene sobre ella. Para el sociólogo francés, superar las dicotomías o dualidades supone estudiar un fenómeno social pensando relacionalmente, "lo real es lo relacional: lo que existe en el mundo social son las relaciones. No interacciones entre agentes o lazos intersubjetivos entre individuos, sino relaciones objetivas que existen 'independientemente de la conciencia o voluntad individual". ${ }^{3}$ En otras palabras, sus conceptos tienen significado gracias a un sistema de relaciones, puesto que un concepto no puede ser utilizado de manera independiente, sino en relación con otro.

Los conceptos de Pierre Bourdieu han sido utilizados en estudios de corte cualitativo y cuantitativo, porque pueden ser construidos empíricamente con ambas metodologías. ${ }^{4}$

Pierre Bourdieu y Loïc Wacquant, Una invitación a la Sociología reflexiva (Buenos Aires: Siglo XIX editores, 2008), 134

4 Bourdieu señala: "Nada se ha explicado, nada se ha comprendido, cuando se ha llegado a establecer la existencia de una fuerte correlación entre una variable llamada independiente y una variable llamada dependiente: mientras no se haya determinado lo que designa en cada caso particular, es decir, en cada relación particular, cada uno de los términos de la relación (por ejemplo, el nivel de instrucción y el conocimiento de los compositores), la relación estadística, por muy grande que sea la precisión que la que pueda determinarse numéricamente, sigue siendo un puro dato, desprovisto de sentido [...]. De esta forma, para interpretar adecuadamente las diferencias observadas [...] será preciso analizar en su totalidad los usos sociales, legítimos o ilegítimos, a los que se presta cada uno". Pierre Bourdieu, La distinción. Criterio y bases sociales del gusto (Madrid: Taurus, 2012), 21.

NOVUM JUS • ISSN: 1692-6013 • Volumen 9 Nº 1 • Enero - Junio 2015 • Págs. 103-122 
Los datos construidos empíricamente para esta investigación posibilitaron observar cómo un agente —el juez — usa el conjunto de sus conocimientos, experiencias y formas de percepción de la realidad para desenvolverse dentro de un espacio específico, que sigue una lógica de operación; ni las estructuras ni los agentes pueden ser entendidos de manera autónoma, porque la relación entre estas y aquellos es lo que permite la explicación de la acción.

Con la reforma penal en México se modificó la disposición jurídica y la acción de sus operadores, observada en las prácticas de estos. El análisis de este cambio mostró cómo se vinculan la estructura y la acción de los jueces en los juicios orales. Se tomó como punto de partida un elemento que ha sido incorporado en la composición del nuevo campo en la lógica de operación de los jueces: la oralidad.

Este cambio paradigmático implicó un cambio en las prácticas de los jueces, lo que me llevó a poner atención en la forma como los operadores jurídicos introyectaron en sus prácticas cotidianas la nueva estructura jurídica, cómo se amoldan los jueces y cómo la modifican.

Podría pensarse que la reforma, por sí misma, cambia el campo del sistema de justicia, pero las acciones de los jueces no lo hacen de modo automático. Bourdieu explica que la acción del agente incide en las estructuras y viceversa, a partir de dimensionar las estructuras objetivas como incorporadas en forma de esquemas de interpretación, a lo que llamará habitus.

Para entender la acción del agente, hay que considerar sus habilidades y los contextos en donde se desarrolla. El concepto de habitus es el que da cuenta de las estructuras objetivas del campo y las incorporadas, que son las elecciones que toman los jueces para modificar sus prácticas. Entre las estructuras objetivas y las prácticas de los agentes no hay una relación de causalidad ni unívoca.

Bourdieu define el campo como:

[...] una red o una configuración de relaciones objetivas entre posiciones. Estas posiciones están objetivamente definidas, en su existencia y en las determinaciones que imponen sobre sus ocupantes, agentes o instituciones, por su situación presente y potencial (situs) en la estructura de distribución de especies del poder (o capital) cuya posesión ordena el acceso a ventajas

110 NOVUM JUS • ISSN: 1692-6013 • Volumen 9 No 1 • Enero - Junio 2015 • Págs. 103-122 
La utilidad práctica de la teoría relacional de Pierre Bourdieu en la investigación...

específicas que están en juego en el campo, así como por su relación objetiva con otras posiciones (dominación, subordinación, homología, etcétera). ${ }^{5}$

El campo es el concepto que da contención al habitus, es decir, es el espacio específico en donde los jueces harán uso de sus capitales. Los capitales son la acumulación de conocimientos y habilidades de los jueces para posicionarse en el nuevo sistema de justicia penal y esta posición tiene impacto en la percepción del resto de los agentes que participan en las audiencias mediante la interacción. En la teoría relacional, los capitales son entendidos como:

Una especie de capital es aquello que es eficaz en un campo determinado, tanto a modo de arma como de asunto de juego en la contienda, que permite a sus poseedores disponer de un poder, influencia, y por tanto existir en el campo en consideración, en lugar de ser considerado una cifra desdeñable. ${ }^{6}$

Los jueces movilizan sus capitales para resignificar sus prácticas a partir de la oralidad que, a su vez, funciona como un capital que adquieren y desarrollan, primero por medio de la capacitación recibida y después por su quehacer cotidiano en las audiencias.

El campo es un pequeño universo social en donde se observan relaciones de poder, relaciones de fuerza, uso de capitales y luchas por transformar o conservar ese espacio, que está dotado de leyes propias que no aplican en otros campos sociales. Pensar en un campo es pensar relacionalmente y en que posee autonomía relativa.

Un campo se construye con regularidades que pueden ser explícitas o no; se configura y opera con reglas específicas escritas y no escritas. Además, puede ser visto como un lugar en donde los jueces hacen uso de sus habilidades y conocimientos.

En el nuevo sistema de justicia penal, los jueces movilizan sus capitales de acuerdo con creencias, habilidades, experiencias previas, intereses y valores, que se manifiestan en su interacción en el campo. Dentro de un campo siempre existirá una relación de fuerzas:

[...] cuya necesidad se impone a los agentes que se han adentrado en él, y como un campo de luchas dentro del cual los agentes se enfrentan, con medios

Bourdieu y Wacquant, Una invitación a la Sociología reflexiva, 135.

Bourdieu y Wacquant, Una invitación a la Sociología reflexiva, 152.

NOVUM JUS • ISSN: 1692-6013 • Volumen 9 Nº 1 • Enero - Junio 2015 • Págs. 103-122 
y fines diferenciados según su posición en la estructura del campo de fuerzas, contribuyendo de este modo a conservar o a transformar su estructura. ${ }^{7}$

Los jueces que se han integrado al sistema de justicia oral han pasado por un proceso de capacitación que los vuelve "profesionales" y que es aplicada con el propósito de renovar sus prácticas y adquirir nuevas habilidades. Sin embargo, no existe una renovación completa de las prácticas, sino una resignificación de ellas.

Con la reforma al sistema de justicia penal se puede observar la reconfiguración del campo jurídico y, por tanto, de los habitus de los jueces. En este proceso tiene gran peso la acumulación de los capitales adquiridos, ya que funcionan como herramientas e instrumentos para la apropiación e interpretación de las nuevas prácticas jurídicas.

En esta investigación fue pertinente iniciar con una reconfiguración del campo del sistema de justicia en México tras la reforma. El campo es la estructura objetiva, pero al mismo tiempo, es un lugar en el que los jueces ponen en práctica todas las habilidades, experiencias previas y conocimientos que poseen y adquieren. Los jueces no solo resignifican sus prácticas a partir del decreto de reforma, sino también de la experiencia que han tenido en su quehacer cotidiano en el poder judicial.

La construcción del campo se hizo desde los datos empíricos obtenidos y, para este caso concreto, por medio del análisis de la reforma, de las entrevistas a los jueces y otros operadores y de la observación de las audiencias. En la construcción del campo contaron tanto las experiencias pasadas de los jueces como su encuentro con el nuevo sistema de justicia oral.

\section{La resignificación de las prácticas de los jueces: el nuevo habitus}

El habitus puede ser un concepto difícil de operacionalizar, debido a su grado de abstracción, por lo que un camino puede ser encontrar el habitus en los capitales de los jueces. Este también contiene las estructuras incorporadas o adquiridas, capitales que pueden apreciarse en los esquemas de interpretación que utilizan los jueces para tomar decisiones o manifestar preferencias. Los esquemas de inter-

7 Pierre Bourdieu, Razones prácticas. Sobre la teoría de la acción (Barcelona: Anagrama, 1997), 49.

112 NOVUM JUS • ISSN: 1692-6013 • Volumen 9 No. 1 • Enero - Junio 2015 • Págs. 103-122 
La utilidad práctica de la teoría relacional de Pierre Bourdieu en la investigación...

pretación se encuentran incorporados en el habitus y por ello es el concepto que da sentido a lo relacional.

La oralidad, para esta investigación, es la práctica incorporada que va a tocar las antiguas prácticas e inercias de los jueces.

Los esquemas de interpretación posibilitaron incluir el material empírico en la investigación y acercarnos al habitus de los jueces: "Bourdieu caracteriza al habitus como un 'sistema de esquemas interiorizados que permiten engendrar todos los pensamientos, percepciones y acciones característicos de una cultura”. ${ }^{8}$

Es importante enfatizar que el habitus es un concepto central, porque reconoce que los individuos participantes no son pasivos y no se limitan a acatar instrucciones. Pierre Bourdieu parte del supuesto de que cada individuo genera expectativas en toda interacción y que tiene intereses que orientan su acción.

El concepto de habitus da cuenta de lo prerreflexivo y también de la intencionalidad presentes en las prácticas de los jueces, es decir, en las prácticas que llevan a cabo de acuerdo con sus esquemas de interpretación que pueden ser producto de sus prácticas pasadas y también de un cálculo estratégico de sus acciones.

La riqueza de utilizar la teoría relacional también evidenció que los jueces tienen resistencias e inercias en sus prácticas con respecto al cambio.

Bourdieu presenta la génesis del habitus como proceso de inculcación de un arbitrario cultural y como incorporación de determinadas condiciones de existencia [...]. La inculcación [... .] supone una acción pedagógica efectuada dentro de un espacio institucional "familiar o escolar" por agentes especializados, dotados de autoridad de delegación, que imponen normas [...]. La incorporación, en cambio, remite a la idea de una interiorización por los sujetos de las regularidades escritas en sus condiciones de existencia [...]. Lo que Bourdieu postula, entonces, es una articulación dialéctica entre inculcación e incorporación, entre lo institucional y la experiencia del mundo social. ${ }^{9}$

Gilberto Giménez, La Sociología de Pierre Bourdieu (México: IIS/UNAM, 1997), 6.

Giménez, La Sociología de Pierre Bourdieu, 11-12.

NOVUM JUS • ISSN: 1692-6013 • Volumen 9 Nº 1 • Enero - Junio 2015 • Págs. 103-122 


\section{Cómo resignifican sus prácticas los jueces en el nuevo campo del sistema de justicia en México}

En este apartado se observará cómo los jueces resignifican sus prácticas y cómo incorporan un nuevo habitus a partir de la reconfiguración del campo del sistema de justica en México y también de sus experiencias pasadas.

Las elecciones que hacen los jueces no son un destino; estas pasan por tamices, entramados y preferencias que se definen por los capitales que poseen y adquieren y la interacción con otros operadores jurídicos.

Al ser un principio generador y organizador de prácticas y representaciones, el concepto de habitus implica observar todos los aspectos de la vida de la persona — sus intereses, conocimientos y experiencias- que definen sus selecciones; es el principio que permite al juez disponer de un conjunto diferenciado de conocimientos y experiencias para resignificar sus prácticas.

El habitus apreciado mediante los capitales muestra que cada juez genera esquemas de interpretación.

En la construcción de la verdad jurídica y en la observación de las nuevas y viejas prácticas encontré un rico terreno de análisis. Ahí confluyen la estructura objetiva — que es el campo del nuevo sistema de justicia, delimitado y establecido- con la reforma y las estructuras incorporadas — que son los conocimientos, las experiencias pasadas y habilidades adquiridas tras la capacitación-. Estas prácticas las incorporan los jueces al momento de llevar a cabo sus funciones dentro de los juicios orales; los capitales viejos y nuevos van conformando un nuevo habitus.

Al utilizar la teoría relacional en la investigación social, no pueden separarse los conceptos clave. Como señala Wacquant:

Los conceptos de habitus y campo le permiten a Bourdieu abandonar los falsos problemas de la espontaneidad personal y la compulsión social, libertad y necesidad, elección y obligación, y dar un paso al estado desde las alternativas comunes de individuo y estructura, micro (Blumer, Coleman) y macroanálisis (Bla, Skocpol) a las que fuerza una ontología social polarizada, dualista: "Uno no tiene que elegir entre estructura y agentes, entre el campo, que hace al

114 NOVUM JUS • ISSN: 1692-6013 • Volumen 9 No 1 • Enero - Junio 2015 • Págs. 103-122 
La utilidad práctica de la teoría relacional de Pierre Bourdieu en la investigación...

significado y valor de las propiedades objetivadas en las cosas o encarnadas en las personas, y los agentes, que juegan con sus propiedades en el espacio de juego así definido", o entre las posiciones entre un espacio de recursos y las urgencias, motivos e "intenciones" socializadas de sus ocupantes. ${ }^{10}$

A continuación se expondrán dos temáticas obtenidas de las entrevistas: ${ }^{11}$ por una parte, la construcción de la verdad jurídica y, por otra, nuevas y viejas prácticas, el sistema "tradicio-oral".

\section{La construcción de la verdad jurídica}

Según el sistema en el que se desenvuelve, la construcción de la verdad jurídica tiene características distintas. En el antiguo sistema se basaba en la prueba circunstancial, o sea, en un pensamiento lógico que los jueces o los secretarios proyectistas desarrollaban a partir de los testimonios desahogados en el proceso. Se privilegiaba la prueba testimonial, en particular la que provenía de los policías y testigos ofrecidos por el Ministerio Público, que era la figura que concentraba todas las funciones, acusaba y casi juzgaba bajo la presunción de culpabilidad.

En las entrevistas se pudo observar que los jueces hacen una resignificación de la práctica de juzgar con base en el trabajo de los secretarios, una labor que ellos desempeñaban en el antiguo sistema.

Recordemos que más de 75\% de los jueces que operan en el estado de Morelos se desempeñó como secretario de los jueces en el antiguo sistema. Se podría decir que los actuales jueces hacen uso de las experiencias del antiguo sistema y de las nuevas prácticas que establece la reforma para construir la verdad jurídica.

Dictar una sentencia al hilo, en el momento que se están recibiendo, que se desarrollaron o se desahogaron las pruebas e inmediatamente tener que dictar una sentencia literalmente de viva voz no es una situación fácil, porque el proyectista se tomaba días. ${ }^{12}$

10 Giménez, La Sociología de Pierre Bourdieu, 49

11 Para identificar las entrevistas, asigné un código de identificación, con el fin de mantener el anonimato de los operadores jurídicos entrevistados: juez, J; abogados defensores, ad; Ministerios Públicos, MP.

12 J2, comunicación personal, 21 de enero de 2014.

NOVUM JUS • ISSN: 1692-6013 • Volumen 9 Nº 1 • Enero - Junio 2015 • Págs. 103-122 
Yo tengo que decretar de manera oral una resolución donde motive en caliente el por qué estoy dándole la razón a una persona y decirle por qué no estoy dándole la razón a la otra y esto requiere ciertas habilidades comunicativas que muchos jueces del sistema tradicional no tenían, porque el sistema no se los exigía. De entrada había un proyectista. ${ }^{13}$

Y también se tenía un secretario proyectista, que es el que analizaba el asunto y proponía un proyecto. Aquí, en estas audiencias, nosotros los jueces no tenemos esos dos grandes brazos. Significa entonces que la gente del MP y la defensa me tienen que dar la información precisa, directa, necesaria, idónea, para que yo no me pierda en el mundo de información que pudiera generarse y poder resolver de acuerdo a las pretensiones de las partes. ${ }^{14}$

En el nuevo sistema, la prueba reina, establecida por la reforma, es la prueba científica o pericial. Se buscó transitar a un sistema garantista basado en el principio de presunción de inocencia; por ello, las pruebas desahogadas deben estar sustentadas científicamente para poder probar el hecho. Sin embargo, los jueces todavía parten de la prueba testimonial para fundamentar sus sentencias.

[... tenemos los testigos de que estos muchachos fueron; lo que sí quedo claro es que fueron por el dinero, ¿no? Y que llegaron en la motocicleta. Yo no veo de dónde, con ese señalamiento directo, yo no necesito más pruebas ni siquiera de la víctima; con esos dos testimonios se puede sostener una sentencia [...] es pura lógica; el proceso es pura lógica. Hay que ver cuestiones formales, por ejemplo, de la acusación [...] entonces, cuando hay ya dos o tres circunstancias que te incriminen, yo con eso puedo hacer una sentencia de condena. ${ }^{15}$

En el análisis de las entrevistas se evidenció que los jueces parten de su experiencia pasada; siguen sentenciando como en el viejo sistema, de manera velada, no explícita y aceptan, en algunos momentos, que siguen utilizando el testimonio para construir la verdad jurídica.

Una de las cosas más cotidianas que hemos tenido sobre este tema es en los delitos de robo [...] es que vengan los policías y digan: "Yo lo detuve" y a lo mejor "lo detuve con las cosas" o "lo detuve porque la víctima me lo señaló".

$13 \mathrm{Jl}$, comunicación personal, 21 de enero de 2014

$14 \mathrm{Jl}$, comunicación personal, 21 de enero de 2014.

15 J5, comunicación personal, 4 de marzo de 2014.

\section{6}

NOVUM JUS • ISSN: 1692-6013 • Volumen 9 Nº 1 • Enero - Junio 2015 • Págs. 103-122 
La utilidad práctica de la teoría relacional de Pierre Bourdieu en la investigación...

¿Habrá alguna duda? Sin embargo, la ley dice que tiene que quedar bien acreditado el delito [...]. De forma indirecta nosotros tenemos la seguridad de que sí participaron, pero no está plenamente acreditado. ¿Qué es lo que llega a ocurrir en estos casos? Adiós, se va. ${ }^{16}$

Dos defensores se expresaron así de la valoración que hacen los jueces:

Pero consideramos que hay pruebas o, más bien, hechos que deberían ser únicamente acreditados por pruebas científicas, como en este caso y hay jueces que dicen: "No me importa la prueba científica; con que lo haya dicho el testigo, es suficiente". ${ }^{17}$

Antes, en el auto de formal prisión, se tenía que acreditar el cuerpo del delito; ahora ya no, ahora nada más que existan indicios que se ha cometido un delito y que está la posibilidad de que el imputado lo cometió. Entonces el estándar probatorio bajó muchísimo; entonces, por puro indicio, te pueden vincular a proceso. ${ }^{18}$

Este fragmento da luz sobre la manera en que, para la construcción de la verdad jurídica, el juez pone una valoración antes del desahogo de la prueba, basado en el testimonio del policía. El testimonio da cuenta de las inercias que los operadores jurídicos tienen en la operación del nuevo sistema. El juez está valorando a partir del esquema de interpretación previo, que domina, porque fue secretario.

Sostuve que la oralidad es un espacio rico de análisis para observar la resignificación de las prácticas de los jueces.

Al final del camino, yo no puedo incidir, porque dentro de las formalidades que yo debo respetar es que soy un ente pasivo, receptor de la información. Entonces yo no puedo subsanar alguna omisión del agente del MP, sobre todo porque ellos son un órgano de carácter técnico y deben de tener estas destrezas para poder comunicar la información y, finalmente, si estoy frente a un MP que no esté debidamente capacitado en esta técnica y destreza, se corre un riesgo muy alto de que el resultado sea negativo cuando deba ser positivo. Entonces, el problema de la implementación no es tanto la infraestructura,

J3, comunicación personal, 4 de marzo de 2014.

ADl, comunicación personal, 13 de marzo de 2014

AD2, comunicación personal, 13 de marzo de 2014

NOVUM JUS • ISSN: 1692-6013 • Volumen 9 Nº 1 • Enero - Junio 2015 • Págs. 103-122 
sino la capacitación de los operadores para generar esas habilidades que en un sistema tradicional no se exigían. ${ }^{19}$

Soso, impersonal, parecía que tratabas con robots o no sé. Desde las preguntas al secretario de acuerdos te impedían que pudieras interrogar libremente a un testigo, porque ya estaba en la declaración, pero ¿qué tal si no lo había dicho? Eso es la libertad probatoria en el desarrollo de las audiencias; es mucho mejor, porque no peleamos contra un papel. El gran paradigma de tener un papel escrito a tener la declaración viva del testigo es que ahora estamos ante, juzgamos a personas. ${ }^{20}$

En estos fragmentos se aprecia que la oralidad incide en la construcción de la verdad jurídica y la valoración de las pruebas. La transición de un sistema a otro evidencia las prácticas y su proceso de resignificación.

Además de la oralidad, se incorporan nuevos elementos que no se encontraban en el viejo sistema, como la interpretación del lenguaje corporal de los involucrados en un proceso.

[... el el papel no te habla, no te expresa, no te dice nada; entonces ahí se queda y [...] si solo valoras el papel, pues no valoras los gestos, la forma de expresarse de la víctima, qué es lo que te quiere dar a entender, porque es la forma en la que platicamos; muchas veces no decimos las palabras correctas, pero por la expresión sabemos que quieren decir las personas. ${ }^{21}$

Como ya mencioné, otra característica del nuevo sistema y que viene con la oralidad es que el juez no conoce previamente de la carpeta de investigación; no conoce los hechos ni las pruebas.

[...] la gran diferencia en este sistema es que nosotros no tenemos nada. Nuestra carpeta administrativa no tiene absolutamente nada, nada más tiene [...] un control de detención ahorita, nada más la indicación de que a mí me asignan el asunto, la petición de audiencia del Ministerio Público que no dice nada del hecho, nada de nada. ${ }^{22}$

\footnotetext{
J1, comunicación personal, 21 de enero de 2014.

J4, comunicación personal, 13 de marzo de 2014

J4, comunicación personal, 13 de marzo de 2014.

J4, comunicación personal, 13 de marzo de 2014.
}

118 NOVUM JUS • ISSN: 1692-6013 • Volumen 9 N No. 1 • Enero - Junio 2015 • Págs. 103-122 
La utilidad práctica de la teoría relacional de Pierre Bourdieu en la investigación...

\section{Nuevas y viejas prácticas, sistema "tradicio-oral"}

De modo somero, pude observar cómo los jueces incorporan un nuevo habitus, que se forma de las experiencias pasadas y las habilidades adquiridas con la capacitación y su experiencia cotidiana en los juicios orales.

Estamos convencidos de que el sistema es bueno; sin embargo, sí le falta mucho que aportarle para que pueda ser como se pretende, que la justicia sea lo más clara posible, porque insisto ante esas deficiencias, considero yo, como defensa, que tiene la Procuraduría [...]. El rol del juez que debería ser nada más, de alguna forma, como el árbitro de esta cuestión del debate del juicio oral; terminan ellos, de alguna manera, este... subsanando sus errores y condenan, a veces sin evidencias y sin las pruebas suficientes. ${ }^{23}$

Tomé la denominación de sistema "tradicio-oral" de los mismos operadores jurídicos. Ellos lo catalogan así, porque consideran que se está haciendo una mezcla de los principios de ambos sistemas. Ello me mostró que los jueces van adoptando y adaptando las nuevas prácticas con sus conocimientos y prácticas previos.

En el nuevo sistema se sigue poniendo especial énfasis en la prueba circunstancial: "[... mezclan cosas del tradicional con cosas del oral [...] así es el 'tradicio-oral'. Aquí le llamamos sistema 'tradicio-oral', mezcla de uno y mezcla de otro y hacemos uno mixto". ${ }^{24}$

[...] porque ellos (los jueces) tienen que dictar una resolución con base en las pruebas que se aportan en el juicio, pero aun así ha habido unas donde nosotras, pues, como defensoras decimos: es que era para una absolutoria. Y ellos tomaron, ahorita está volviendo a resolver con la prueba circunstancial. ${ }^{25}$

En la práctica, los jueces hacen uso de sus dos conocimientos: el adquirido en sus experiencias previas dentro del poder judicial y el que adquieren a partir de lo establecido en la reforma; con ello, forman un híbrido del sistema en lo que denominan el sistema "tradicio-oral".

AD1, comunicación personal, 13 de marzo de 2014

J4, comunicación personal, 13 de marzo de 2014.

AD1, comunicación personal, 13 de marzo de 2014

NOVUM JUS • ISSN: 1692-6013 • Volumen 9 Nº 1 • Enero - Junio 2015 • Págs. 103-122 


\section{Conclusión}

Como lo señala Loïc Wacquant, manufacturar los conceptos en las investigaciones sociojurídicas significa asumir que:

La dificultad peculiar de la sociología, entonces, es producir una ciencia precisa a partir de una realidad imprecisa, difusa y embrollada. Para ello es mejor que sus conceptos sean polimorfos, flexibles y adaptables, en lugar de definidos, calibrados y de rígida aplicación. ${ }^{26}$

El uso de Bourdieu en la investigación empírica, más allá de los debates alrededor de su obra, de su validez o de su falacia, permitió comprender el sentido de superar las dicotomías más usadas en la Sociología, así como entender que los conceptos son flexibles y deben construirse a lo largo de la investigación y de la delimitación metodológica. La fortaleza de esta propuesta también consiste en que la investigación sociológica es una construcción teórico-metodológica y que se realiza de manera simultánea.

En el caso concreto de esta investigación sobre los juicios orales, los conceptos de Bourdieu me permitieron observar el tránsito y la transformación del campo del sistema de justicia y cómo las prácticas de los jueces son resignificadas en el nuevo campo y transitan hacia la construcción de un nuevo habitus, en la cual incorporan esquemas de interpretación de sus experiencias previas en el antiguo sistema, nuevas habilidades y nuevos esquemas de interpretación. En sus palabras, construyen con lo viejo y lo nuevo un sistema de justicia mixto: el "tradicio-oral" y reconocen abiertamente que la nueva estructura del sistema penal está tamizada por sus viejos esquemas de interpretación.

La teoría relacional llevó a entender que las estructuras no se imponen al agente, sino que este las modifica, las amolda y las adapta para hacer elecciones dentro del campo en donde se encuentra.

Tras el análisis de las entrevistas a los operadores jurídicos, se apreció que los jueces siguen poniendo especial énfasis en la construcción de la verdad jurídica por medio de un esquema de interpretación basado en pruebas testimoniales; no

26 Bourdieu y Wacquant, Una invitación a la Sociología reflexiva, 49. 
La utilidad práctica de la teoría relacional de Pierre Bourdieu en la investigación...

obstante, en ciertos momentos logran equilibrar sus antiguas prácticas con las nuevas habilidades adquiridas.

Con este primer acercamiento a la resignificación de las prácticas de los jueces y de la restructuración del campo de justicia en materia penal, no solo se identificó el desarrollo de las habilidades y los conocimientos de los jueces, sino su impacto en la forma de administrar justicia.

El efecto de la implementación de la reforma en todo el país es un proceso gradual y lento, porque deben modificarse las prácticas de los operadores del sistema, así como la estructura y las funciones de las instituciones del poder judicial mexicano. Conocer el verdadero resultado que tendrá la restructuración del sistema de justicia en México será un largo proceso. Con la teoría relacional podemos ver cómo va surgiendo este cambio: el del sistema de justicia y el de los habitus de los operadores jurídicos en México.

\section{Referencias}

Bourdieu, Pierre. La distinción. Criterio y bases sociales del gusto. Madrid: Taurus, 2012.

Bourdieu, Pierre. Razones prácticas. Sobre la teoría de la acción. Barcelona: Anagrama, 1997.

Bourdieu, Pierre y Loïc Wacquant. Una invitación a la Sociología reflexiva. Buenos Aires: Siglo XIX editores, 2008.

Giménez, Gilberto. La Sociología de Pierre Bourdieu. México D. F.: IIS/UNAM, 1997.

Consejo de la Judicatura Federal. "El nuevo sistema de justicia penal acusatorio, desde la perspectiva constitucional". http://www.cjf.gob.mx/reformas/documentos/ElnuevosistemadeJusticiaPenalAcusatorio.pdf (acceso noviembre 2, 2014).

Consejería Jurídica del Poder Ejecutivo del Estado de Morelos. Código de Procedimiento Penal para el estado de Morelos. Cuernavaca: Periódico Oficial Tierra y Libertad $\mathrm{N}^{\circ}$ 4570, 22 de noviembre de 2007.

Secretaría de Gobierno. Decreto por el que se reforman y adicionan diversas disposiciones de la Constitución Política de los Estados Unidos Mexicanos. México D. F: Diario Oficial de la Federación, 18 de junio de 2008.

Cámara de Diputados. Dictamen de las Comisiones Unidas de Puntos Constitucionales y de Justicia, con Proyecto de Decreto que reforma, adiciona y deroga diversas disposiciones de la Constitución Política de los Estados Unidos Mexicanos. México D. F: Gaceta Parlamentaria de la Cámara de Diputados, año XI, No. 2401-VIII, 11 de diciembre de 2007.

NOVUM JUS • ISSN: 1692-6013 • Volumen 9 Nº 1 • Enero - Junio 2015 • Págs. 103-122 
Gobierno del estado de Morelos. "Plan estatal de desarrollo 2007-2012 del estado de Morelos". http://www.transparenciamorelos.mx/sites/default/files/PlanEstatal-2007-2012_0. pdf (acceso agosto 1, 2014).

Poder Judicial del Estado de Morelos. "Información en Poder Judicial del Estado de Morelos, Juzgados de primera instancia”. http://tsjmorelos2.gob.mx/juicios_orales. html\#acuerdos2010 (acceso septiembre 19, 2014). 